PROCEEDINGS OF THE

AMERICAN MATHEMATICAL SOCIETY

Volume 134, Number 4, Pages 989-993

S 0002-9939(05)08247-X

Article electronically published on September 28, 2005

\title{
LINEAR SERIES OVER REAL AND $p$-ADIC FIELDS
}

\author{
BRIAN OSSERMAN
}

(Communicated by Michael Stillman)

\begin{abstract}
We note that the degeneration arguments given by the author in 2003 to derive a formula for the number of maps from a general curve $C$ of genus $g$ to $\mathbb{P}^{1}$ with prescribed ramification also yields weaker results when working over the real numbers or $p$-adic fields. Specifically, let $k$ be such a field: we see that given $g, d, n$, and $e_{1}, \ldots, e_{n}$ satisfying $\sum_{i}\left(e_{i}-1\right)=2 d-2-g$, there exists smooth curves $C$ of genus $g$ together with points $P_{1}, \ldots, P_{n}$ such that all maps from $C$ to $\mathbb{P}^{1}$ can, up to automorphism of the image, be defined over $k$. We also note that the analagous result will follow from maps to higher-dimensional projective spaces if it is proven in the case $C=\mathbb{P}^{1}, n=3$, and that thanks to work of Sottile, unconditional results may be obtained for special ramification conditions.
\end{abstract}

\section{Statements And COntext}

Let $k$ be either the real numbers, or a finite extension of $\mathbb{Q}_{p}$ for some $p$. The purpose of this note is to discuss what results may be obtained over $k$ from the arguments of [5, which a priori give results only over $\bar{k}$.

For our first two results, we will fix integers $g, d, n$, and $e_{1}, \ldots, e_{n}$ satisfying $\sum_{i}\left(e_{i}-1\right)=2 d-2-g$. Given this constraint and a smooth curve $C$ of genus $g$ with points $P_{1}, \ldots, P_{n}$, we have a finite expected number of maps to $\mathbb{P}^{1}$ of degree $d$ which are ramified to order at least $e_{i}$ at $P_{i}$ for every $i$, up to automorphism of the image. Note that when $g>0$, each of these maps will have some additional ramification beyond that which is specified. The approach of [5] was to use a degeneration argument involving the Eisenbud-Harris theory of limit linear series to produce a formula for the number of such maps over $\bar{k}$ when $C$ and the $P_{i}$ are taken to be general, and to show that in this case, all maps are without multiplicities. In this note, we show that the same arguments further imply:

Corollary 1.1. There exists a smooth curve $C$ over $k$ of genus $g$ together with $k$-points $P_{1}, \ldots, P_{n}$ on $C$ such that every $\bar{k}$-morphism from $C$ to $\mathbb{P}^{1}$ of degree $d$ and ramified to order at least $e_{i}$ at $P_{i}$ for every $i$ is multiplicity-free and can, after possible composition with a $\bar{k}$-automorphism of the image, be defined over $k$ itself.

A closer examination of the degeneration arguments yields:

Corollary 1.2. If further $g \leq 1$, then the curve $C$ of Corollary 1.1 may be specified arbitrarily, and the conclusion holds whenever the $P_{i}$ are sufficiently close together.

Received by the editors March 20, 2004 and, in revised form, November 7, 2004.

2000 Mathematics Subject Classification. Primary 14H51, 14P99.

(C)2005 American Mathematical Society 
Note that in the case of $g=0$ and $k=\mathbb{R}$, these results were previously known by work of Sottile (see [7, Thm. 1]); see also the remarks on the Shapiro-Shapiro conjecture below for a much stronger result due to Eremenko and Gabrielov.

The same generalizations as in [5] to maps to higher-dimensional projective spaces also apply. Because ramification terminology is more standard in the context of linear series, we phrase our result in that language. Keeping $g, d$, and $n$, we also suppose that we have an integer $r$, and now $n$ vanishing sequences $a^{i}:=a_{0}^{i}, \ldots, a_{r}^{i}$ satisfying $\sum_{i, j}\left(a_{j}^{i}-j\right)=(r+1)(d-r)-r g$. As in [5], in the higher-dimensional case the base case of three points on $\mathbb{P}^{1}$ remains open, and our result is therefore conditional:

Corollary 1.3. Suppose that for a given $d$ and $r$ and arbitrary vanishing sequences $a^{1}, a^{2}, a^{3}$ satisfying $\sum_{i, j}\left(a_{j}^{i}-j\right)=(r+1)(d-r)$, every $\bar{k}$-linear series of degree $d$ and dimension $r$ on $\mathbb{P}^{1}$ with vanishing sequences $a^{1}, a^{2}, a^{3}$ at 0,1 , and $\infty$ respectively, is multiplicity-free and defined over $k$. Then for the same $d$ and $r$, and any $g, n$, and $a^{i}$ as above, there exists a smooth curve $C$ over $k$ of genus $g$ and $n k$-points $P_{1}, \ldots, P_{n}$ of $C$ such that every linear series of degree $d$ and dimension $r$ on $C$, which has vanishing sequence at least $a^{i}$ at $P_{i}$ for every $i$, is multiplicity-free and defined over $k$.

In the case that $g=0$ and $k=\mathbb{R}$, this is closely related to, but substantially weaker than, the Shapiro-Shapiro conjecture (see [8, Conj. 3.1]), which asserts that zero-dimensional intersections of Schubert cycles associated to osculating flags at distinct real points of the rational normal curve should consist entirely of real points. Indeed, a stronger version of this conjecture asserts that these points should be multiplicity-free (see [7, Conj. 5]). Note that this is substantially stronger than the situation for an algebraically closed field, where one will have multiplicities for certain special configurations of points, even when they remain distinct. Eremenko and Gabrielov proved the full reality conjecture in the case $r=1$ in [3], thereby providing a much stronger result than Corollaries 1.1 and 1.2 in that specific case.

In particular, we note that the condition required in Corollary 1.3 is, in the case $k=\mathbb{R}$, a special case of the Shapiro-Shapiro conjecture, which is not only widely believed to be true, but also supported by extensive experimental data in that case. The results presented here therefore provide some substantial data on the question of what the anticipated behavior might be in the higher-genus case, where expectations are not as well established.

However, since we cannot yet establish the base case of Corollary 1.3. we observe that thanks to work of Sottile ([7]) handling the $g=0$ case, when $k=\mathbb{R}$ we can obtain an unconditional form of Corollary 1.3 for special ramification conditions. Specifically:

Corollary 1.4. Given $d, r, g, n$ and $a^{i}$ as in Corollary 1.3 , suppose that $k=\mathbb{R}$ and all but at most two of the $a^{i}$ are special; that is, they are each either of the form $0,0, \ldots, 0, a$ or $0, a, a+1, \ldots, a+r-1, a+r$ for some a which is allowed to vary with $i$. Then there exists a smooth curve $C$ over $k$ of genus $g$ and $n k$-points $P_{1}, \ldots, P_{n}$ of $C$ such that every linear series of degree $d$ and dimension $r$ on $C$, which has vanishing sequence at least $a^{i}$ at $P_{i}$ for every $i$, is multiplicity-free and defined over $k$.

Finally, we make some observations on the $p$-adic case which have some indirect bearing on the real case as well. 
Remark 1.5. In very simple cases of Corollary 1.1 such as $g=0, d=3, n=4$, $e_{i}=2$, it is possible to solve for the maps explicitly, in closed form. In doing so, one sees that the rationality behavior of the real case does not carry over to the $p$-adic case. Instead, one has certain configurations of points where the maps are defined over the base field, and certain configurations where they are not. This immediately implies that arguments as general as those presented here, which use no properties of the real numbers not also satisfied by the $p$-adic fields, cannot prove rationality results as strong as the Shapiro-Shapiro conjecture.

On the other hand, this may not present such a substantial limitation, as one need not look so far beyond the ideas discussed here to find properties distinguishing the real case. For instance, because real intervals are connected, in the real case one can conclude that if on an interval of configurations one always has that the maps are all multiplicity-free, and that they are all real at a single point in the interval, then they are all real on the entire interval. In contrast, the $p$-adic interval is totally disconnected, and the same assertion fails in this setting, as can be verified directly in the same simple case discussed above.

\section{Proofs}

The arguments given here are not intended to be self-contained, but rather serve only to explain how the degeneration argument of [5] yields the asserted corollaries. We begin with some general remarks on the validity of the constructions used in [5] over non-algebraically closed fields.

First, the construction of appropriate families of curves with specified special fiber in Winters' 9 does not in fact require the base field to be algebraically closed. Indeed, standard deformation theory will produce a regular formal surface as in the hypothesis of [9, Prop. 1.1]. In the argument there, one need only note that while the Artin algebraization step requires an étale base change, this base change occurs within the henselization of the local ring of the base rather than the strict henselization, so will not extend the residue field. Similarly, we need to know that we can construct our family to have sections through given smooth points of the special fiber. This follows because the étale base changes used in constructing sections through smooth points in the argument in [1, Prop. 2.2.14] are obtained as closed subschemes of the family through the smooth point in question, and in particular do not extend the residue field.

Next, there is the question of using the theory of limit linear series when over a non-algebraically closed field. While the author sees no obstruction to the original Eisenbud-Harris construction in 2 going through in such generality, the concerned reader may also refer to the alternative, more functorial construction for the main theorems given in [4, Ch. II], which is phrased throughout for more general bases.

With these comments out of the way, we remark on the basic property of real and $p$-adic fields that will allow us to draw our corollaries over such $k$. Namely, suppose $f: X \rightarrow Y$ is a finite $k$-morphism of schemes over $k$, and $Y$ is in fact a smooth variety over $k$. Let $y \in Y(k)$ be a $k$-valued point of $Y$, and suppose that $X_{y}$ is reduced, and consists entirely of points with residue field $k$. Then it follows from the inverse function theorem (see [6. Thm., p. 89 and Thm. 2, p. 83] for the non-Archimedian setting) that there is a neighborhood of $y$ in the analytic topology on $Y$ inside which each fiber also consists of reduced points defined over $k$. 
We thus have:

Proof of Corollaries 1.1 and 1.3 . The arguments are precisely those of Theorems 2.5 and 2.6 of [5]. One begins by deriving the conditional statement of Corollary 1.3 inductively, first concluding the desired statement for arbitrary numbers of points on $\mathbb{P}^{1}$, and then using a comb curve degeneration to obtain the result for curves of higher genus. As we have seen, the constructions are not a problem over non-algebraically closed fields, and the only modification is that our conclusions about rationality, rather than holding in a Zariski neighborhood of the special fiber at each step in the induction, hold only in an analytic neighborhood. Hence, we settle for the weaker statement of existence of curves and configurations of points. We also note for the higher-genus case that in the argument treating the elliptic tails in the proof of Theorem 2.5, the conclusion that the space of linear series in question is a single reduced point also implies that the point is defined over $k$, since otherwise it would no longer be a single point after passing to $\bar{k}$.

The second step is to conclude the unconditional $r=1$ statement of Corollary 1.1. As with the treatment of the elliptic tails, in the base case of three points on $\mathbb{P}^{1}$ for $r=1$, where the intersection number is 1 , it follows that the point is defined over $k$, or it would not remain a single point upon passing to $\bar{k}$.

We also describe:

Proof of Corollary 1.2. For $g=0$, we immediately see that the nature of the degeneration argument used above is precisely that all linear series will be rational when points are sufficiently close together; in fact, two points are allowed to be arbitrary, and the rest will be close together. The idea for $g=1$ is simply to specialize the higher-genus argument above to a family which is constant away from the special fiber. If we start with an arbitrary curve $E$ of genus 1 over $k$, we can fix any $k$-valued point 0 on it, with which we make $E$ into an elliptic curve over $k$. Now, consider the constant family $E \times{ }_{k} E$ over $E$, and blow up the point corresponding to $(0,0)$. This blow-up introduces a rational component in the fiber above 0 . We can choose $n$ sections specializing to arbitrarily close points on this rational component by the general theory above. However, we can do so constructively and without any base change by taking our sections to be sufficiently high multiples of the diagonal imbedding. We can then apply our limit linear series argument as above to this generically constant family to conclude the desired statements on $E$.

Finally, we have:

Proof of Corollary 1.4. The proof proceeds precisely as in obtaining the highergenus case of Corollary 1.3 from the genus 0 case. The main observation is that the new vanishing sequences of the form $0,2,3, \ldots, r, r+1$ introduced on the rational component of the special fiber at the nodes are also special Schubert conditions, so that if we have the desired result for $g=0$, it follows for higher $g$. Lastly, $g=0$ is a theorem of Sottile; see [7, Thm. 1].

Remark 2.1. Using the same techniques, one can obtain a new proof of the $g=0$ case of Corollary 1.4 in much the same spirit of Sottile's proof, but using the machinery of limit linear series rather than results on decomposition of limits of cycle intersections. Indeed, the main observation in the proof of [7, Thm. 1] is that in the three-point case, if at least one ramification condition is special, the 
intersection number is 1 , and we automatically obtain transversality and rationality. Using this and the degeneration argument for the $g=0$ case of Corollary 1.3, we recover Sottile's result.

\section{ACKNOWLEDGEMENTS}

I thank Frank Sottile for bringing my attention to the reality questions and describing the current state of knowledge, as well as suggesting Corollaries 1.2 and 1.4. I would also like to thank Max Lieblich and Brian Conrad for their helpful conversations on relevant background results over non-algebraically closed fields.

\section{REFERENCES}

1. Siegfried Bosch, Werner Lutkebohmert, and Michel Raynaud, Neron models, Springer-Verlag, 1991. MR1045822(91i:14034)

2. David Eisenbud and Joe Harris, Limit linear series: Basic theory, Inventiones Mathematicae 85 (1986), 337-371. MR0846932 (87k:14024)

3. A. Eremenko and A. Gabrielov, Rational functions with real critical points and the B. and $M$. Shapiro conjecture in real enumerative geometry, Annals of Mathematics 155 (2002), no. 1, 105-129. MR.1888795 (2003c:58028)

4. B. Osserman, Limit linear series in positive characteristic and Frobenius-unstable vector bundles on curves, Ph.D. thesis, MIT.

5. - The number of linear series on curves with given ramification, International Mathematics Research Notices 2003, no. 47, 2513-2527. MR2007538 (2004g:14011)

6. J. P. Serre, Lie algebras and Lie groups, second ed., Lecture Notes in Mathematics, no. 1500, Springer-Verlag, 1992. MR1176100 (93h:17001)

7. Frank Sottile, The special Schubert calculus is real, Electronic Research Announcements of the AMS 5 (1999), 35-39. MR1679451 (2000c:14074)

8. - Real Schubert calculus: Polynomial systems and a conjecture of Shapiro and Shapiro, Experimental Mathematics 9 (2000), no. 2, 161-182. MR1780204 (2001e:14054)

9. Gayn B. Winters, On the existence of certain families of curves, American Journal of Mathematics 96 (1974), no. 2, 215-228. MR0357406 (50:9874)

Department of Mathematics, University of California Berkeley, Berkeley, CaliforNIA $94720-3840$ 